Supporting Information for

\title{
Directed Self-Assembly of Triblock Copolymer on Chemical Patterns for Sub-10-nm Nanofabrication Via Solvent Annealing
}

Shisheng Xiong, ${ }^{\dagger}$ Lei Wan, ${ }^{*}$ Yoshihito Ishida, ${ }^{\dagger \mid}$ Yves-Andre Chapuis, ${ }^{\not 1}$ Gordon S. W. Craig, ${ }^{\dagger}$ Ricardo Ruiz, ${ }^{\star}$ Paul F. Nealey*广

${ }^{\dagger}$ Institute for Molecular Engineering, University of Chicago, IL, 60637, United States

*HGST, a Western Digital company, San José, CA, 95135, United States

"Present address: Department of Chemistry, Faculty of Engineering, Kanagawa University

Tokyo, Japan

${ }^{\S}$ Present address: Lam Research Corporation, Fremont, CA, 94538, United States

Corresponding email address: nealey@uchicago.edu 


\section{Description of Fluctuation Equations Used to Calculate $\chi_{E f f}(\phi, T)$ and $\phi_{\text {Odt }}$}

The calculation of $\phi_{\mathrm{ODT}}$ was accomplished by determining $\chi_{\text {eff }}(\phi, T)$ and setting it equal to the standard value of $\left(\chi^{N}\right)_{\mathrm{ODT}}$, which is 10.495 and 19 for symmetric diblock and triblock copolymers, respectively. The fluctuation effects can change $(\chi N)_{\mathrm{ODT}}$, as shown by Fredrickson and Helfand for block copolymers in the melt: ${ }^{1}$

$$
(\chi N)^{\prime}{ }_{O D T}=(\chi N)_{O D T}+41.022 N^{\prime-0.333}
$$

Here, $N^{\prime}=N \beta^{6} / v^{2}$, where $N$ is the degree of polymerization, $\beta$ is the statistical segment length, and $v$ is the monomer volume. For our calculations we used $\beta=0.75 \mathrm{~nm}$, and $v=1.64 \mathrm{x}$ $10^{-22} \mathrm{~cm}^{3}$. Also, in this work the triblock copolymers were not truly symmetric, having a PS block volume fraction of 0.55 . Therefore, we used 19.75 for $(\chi N)_{\text {ODT }}$ of the triblock copolymer.

For block copolymers in a nonselective solvent in the concentrated regime, $\chi$ should be

replaced with $\chi_{\text {eff. }}{ }^{2}$ The dependence of $\chi$ on $T$ is given by Equation 3 in the manuscript. Taking Equations 1 and 3 in the manuscript into account, the complete expression for $\chi_{e f f} N$ for PS- $b$-P2VP is:

$$
\chi_{e f f} N=\left(\frac{63}{T}-0.033\right) \phi^{\frac{\alpha}{6}} N
$$

where $\alpha$ is the power factor for the dilution approximation (determined in Figure 2b). By equating the right sides of equations $\mathrm{S} 1$ and $\mathrm{S} 2, T_{\mathrm{ODT}}$ can be found:

$$
T_{\mathrm{ODT}}=\frac{63 \phi^{\frac{\alpha}{6}} N}{(\chi N)_{\mathrm{ODT}}+0.033 \phi^{\frac{\alpha}{6}} N} .
$$

By setting $T_{\mathrm{ODT}}$ to $25{ }^{\circ} \mathrm{C}$ and numerically solving for $\phi$, $\phi_{\mathrm{ODT}}$ could be determined. Fredrickson and Leibler derived the following equation for symmetric block copolymers in the semidilute regime, in which the effect of fluctuation on $(\chi N)_{\mathrm{ODT}}$ depends on $\phi^{2}$

$$
\left(\chi_{e f f} Z\right)_{\mathrm{ODT}}=(\chi N)_{\mathrm{ODT}}+41 Z^{-\frac{1}{3}}
$$

where 


$$
Z=N \phi^{\frac{1}{3 v-1}}
$$

and $v=0.589$. From Fig. $2 \mathrm{~b}$ and Equation 1 in the manuscript, $\chi$ eff $=\chi \phi^{0.207}$. Combining this with Equation S5 gives $\left(\chi_{e f f} Z\right)_{\mathrm{ODT}}=\chi \phi^{1.511}$. Substituting $\left(\chi_{\text {eff }} Z\right)_{\mathrm{ODT}}=\chi \phi^{1.511}$ into Equation S4 and using the temperature dependency of $\chi$ provided by Equation 3 in the manuscript yields.

$$
T_{\mathrm{ODT}}=\frac{63 \phi^{1.511} N}{\left[(\chi N)_{\mathrm{ODT}}+41 N^{-0.333} \phi^{-0.435}\right]+0.033 \phi^{1.511} N} .
$$

As with Equation S3, Equation S6 could be solved numerically to determine $\phi_{\mathrm{ODT}}$ with $T_{\mathrm{ODT}}=$ $25{ }^{\circ} \mathrm{C}$.

\section{Figures}

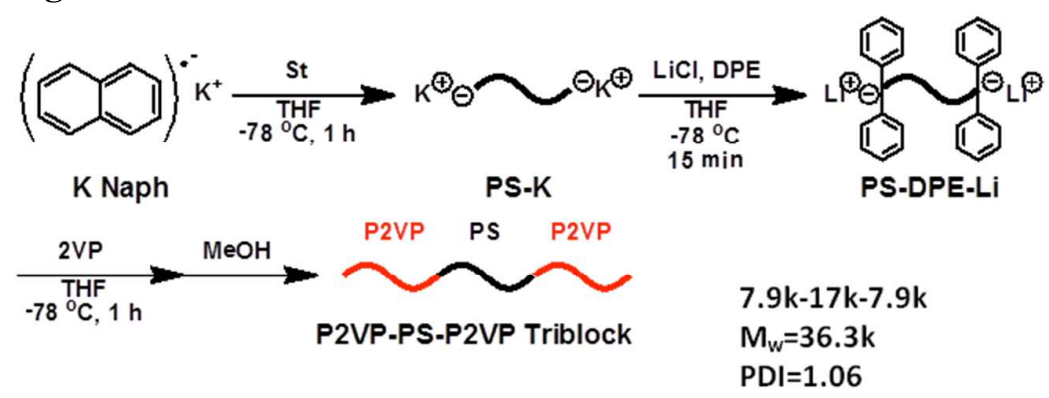

Figure S1. Overview of the copolymerization method used to synthesize P2VP- $b$-PS- $b$-P2VP (ABA linear) triblock copolymer. The molecular formula, molecular weight, and polydispersity of symmetrical P2VP- $b$-PS- $b$-P2VP are listed. 

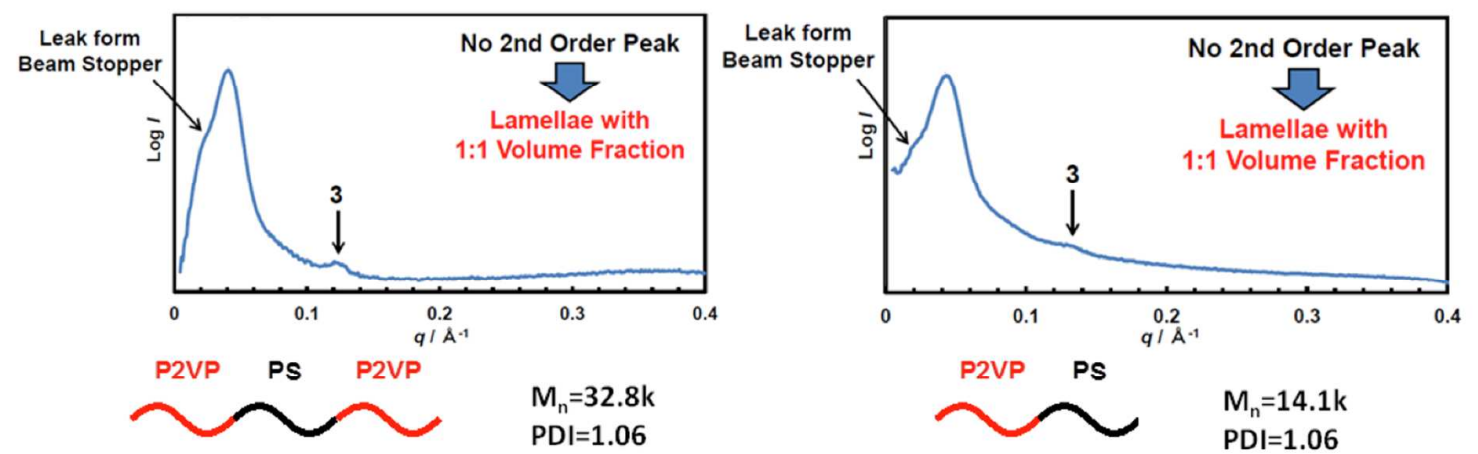

Figure S2. Small angle X-ray scattering scans determined that both the triblock and diblock copolymer are microphase separated at $150{ }^{\circ} \mathrm{C}$.

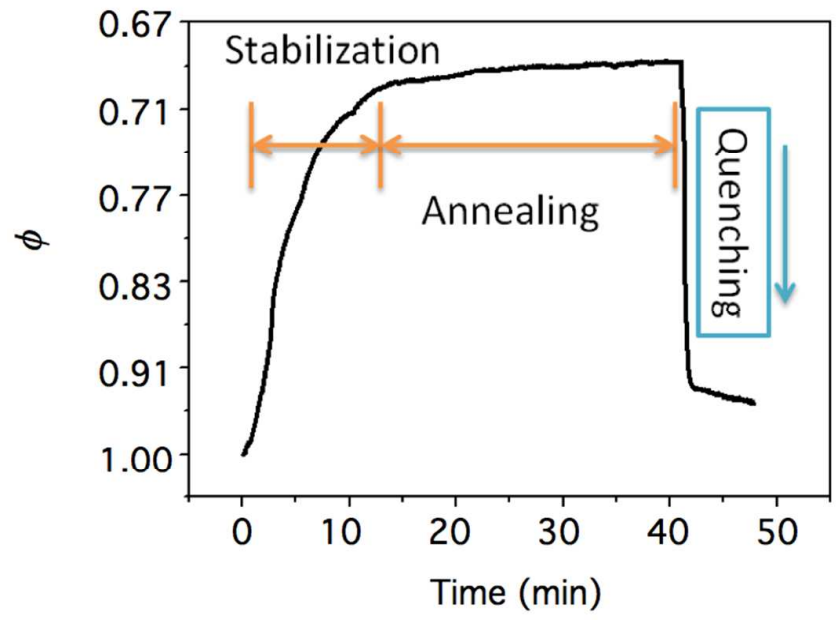

Figure S3. Typical swelling curve, expressed in polymer fraction $\phi$ of solvated film, for the solvent annealing of triblock copolymers in the flow chamber. The film thickness measurement was taken once every three seconds. 


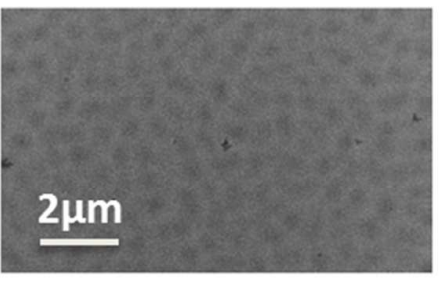

On $40 \%$ PS

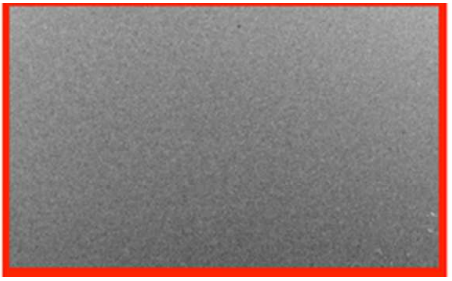

On $65 \%$ PS

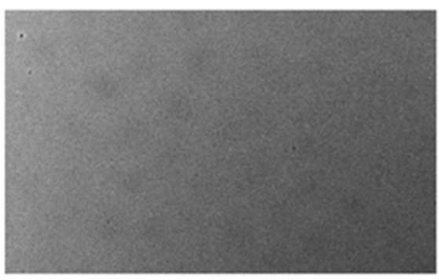

On $100 \%$ PS

Figure S4. Macro-scale SEM images show that the neutral window for acetone annealing of triblock copolymer, in terms of styrene content of a random copolymer brush on the underlying substrate, for acetone annealing of triblock copolymer is centered at $\sim 65 \%$ of styrene by weight. However, the neutral window proved to be quite asymmetric and broad since no apparent hole/islands appear even on $100 \%$ PS surface. The scale bar applies to all images. 
a
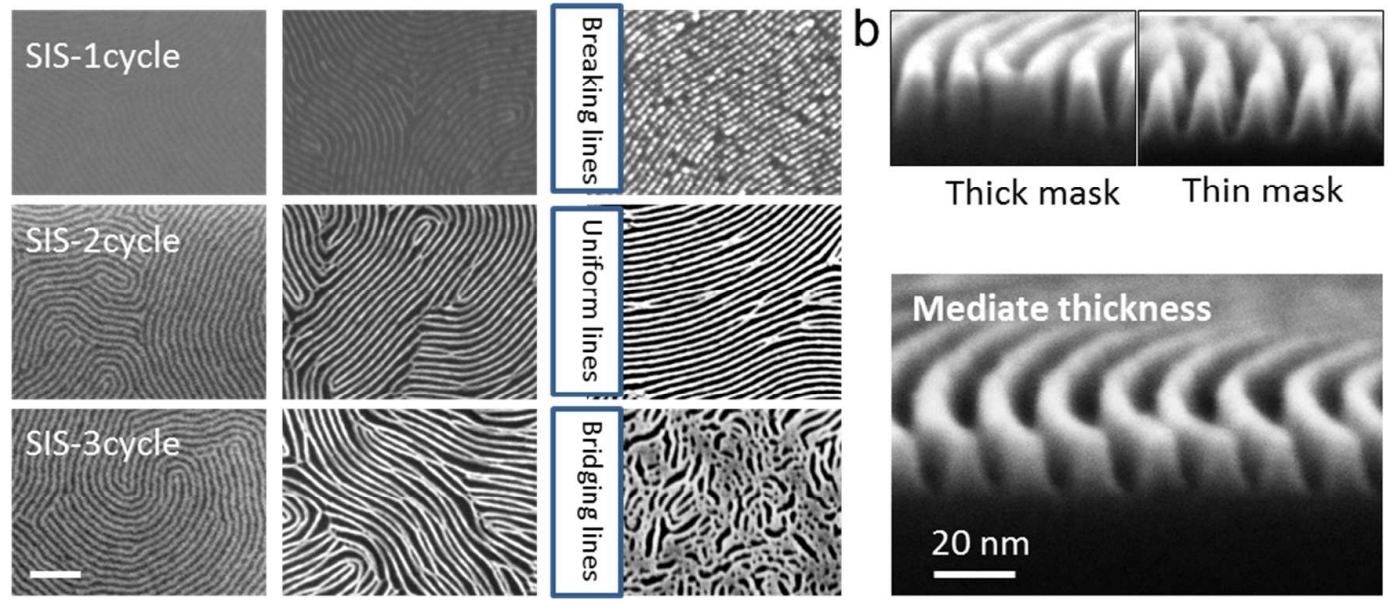

Thick mask

Thin mask

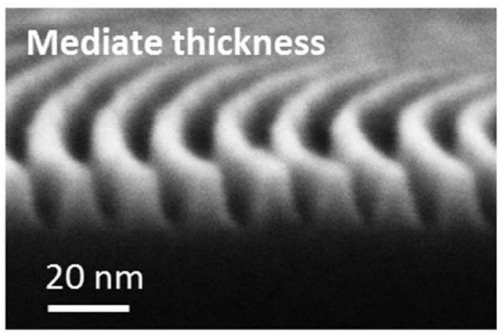

C
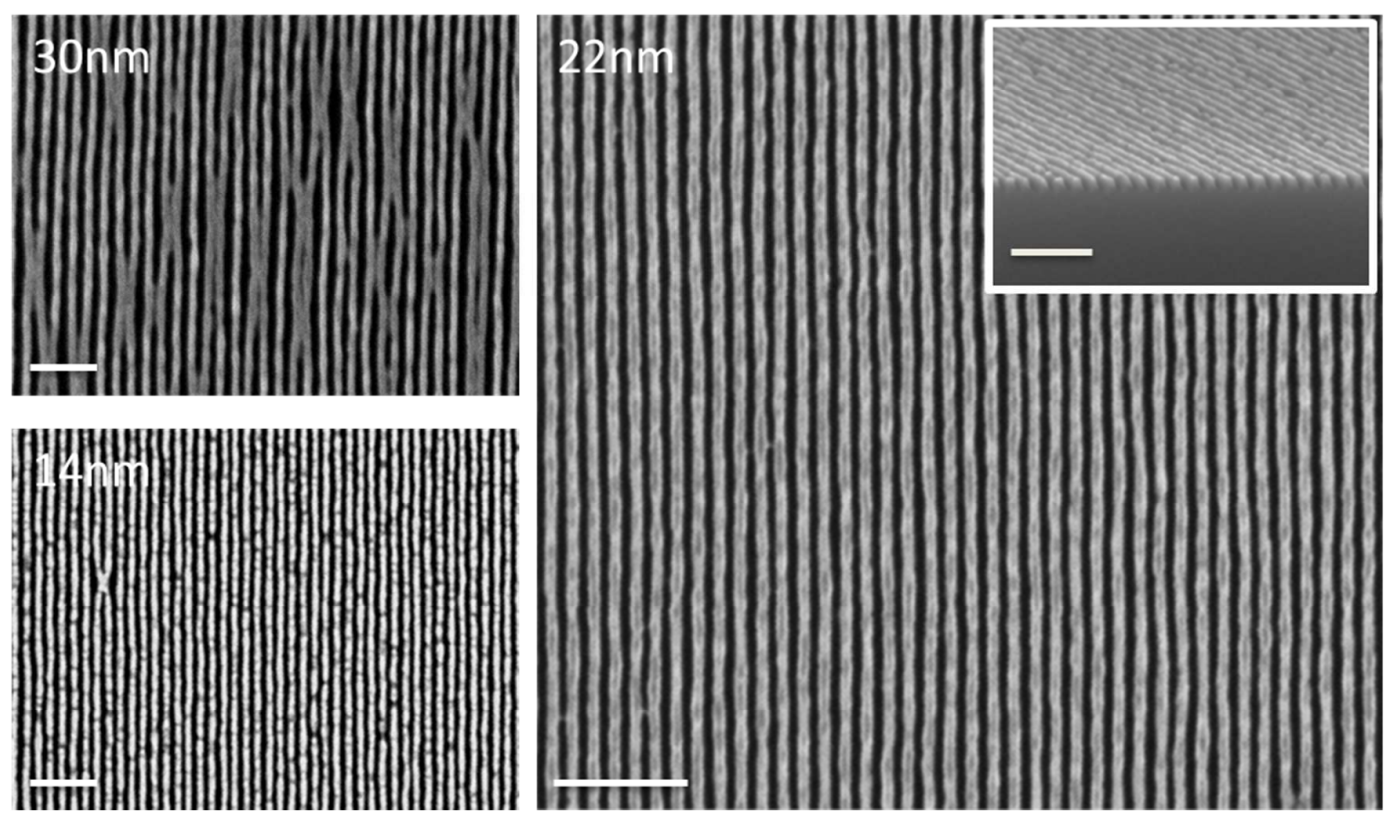

Figure S5. Analysis of sequential infiltration synthesis (SIS) process and triblock copolymer (VSV-33) film thickness on etching of underlying silicon. a) Results of 1, 2, and 3 cycles of the SIS process. From left to right: top-down SEM images of self-assembled BCP, top-down and cross-section SEM images of $\mathrm{AlO}_{\mathrm{x}}$ lines after SIS, and top-down and cross-section SEM images of etched silicon. b) Effect of BCP film thickness. A thick film (30 nm) leads to collapse and a thin film (14 nm) leads to over etching. At the intermediate mask thickness (22 $\mathrm{nm})$, the pattern transfer generated vertical sidewall profile in the Si layer. c) Top-down of patterns etched in silicon using a mask made from directed assembly of three different thicknesses of VSV-33 film (14, 22, and $30 \mathrm{~nm})$ and subsequent $\mathrm{AlO}_{\mathrm{x}}$ formation via 2 cycles of 
the SIS process. The cross-section SEM image in the inset showed the final result of pattern transfer with the 22-nm-thick film. All unlabeled scale bars correspond to $100 \mathrm{~nm}$.

\section{REFERENCES}

1. Fredrickson, G. H.; Helfand, E. Fluctuation Effects in the Theory of Microphase Separation in Block Copolymers. J. Chem. Phys. 1987, 87, 697-705.

2. Fredrickson, G. H.; Leibler, L. Theory of Block Copolymer Solutions - Nonselective Good Solvents. Macromolecules 1989, 22, 1238-1250. 
Table of Contents Graphic

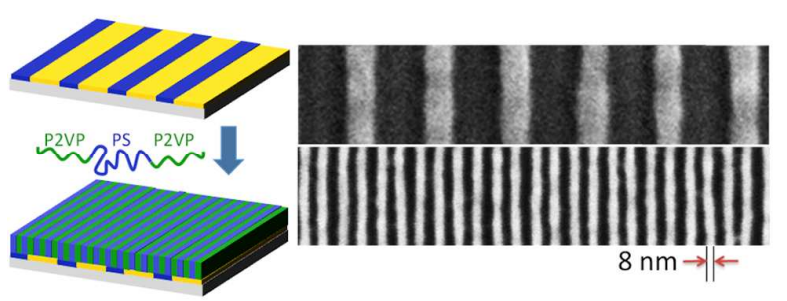

